\title{
Measuring Tissue Properties and Monitoring Therapeutic Responses Using Acousto-Optic Imaging
}

\author{
Todd W. Murray, ${ }^{1}$ Puxiang Lai, ${ }^{2}$ and Ronald A. Roy ${ }^{3}$ \\ ${ }^{1}$ Department of Mechanical Engineering, University of Colorado at Boulder, Boulder, CO, USA; ${ }^{2}$ Department of Biomedical \\ Engineering, Washington University in St. Louis, St. Louis, MO, USA; and ${ }^{3}$ Department of Mechanical Engineering, \\ Boston University, Boston, MA, USA \\ ( Received 22 July 2011; accepted 29 September 2011)
}

Associate Editor Daniel Elson oversaw the review of this article.

\begin{abstract}
Acousto-optic imaging is a hybrid imaging technique that exploits the interaction between light and sound to image optical contrast at depth in optically turbid media with the high spatial resolution of ultrasound. Quantitative measurement of optical properties using this technique is confounded by multiple parameters that influence the detected acousto-optic signal. In this article, we describe the origin of the acousto-optic response and review techniques that have been proposed to relate this response to the optical properties of turbid media. We present an overview of two acousto-optic sensing approaches. In the first, we demonstrate that the local transport mean free path within turbid media can be obtained by varying the pressure of the ultrasound field and processing the resulting acousto-optic signals. In the second, we demonstrate that the acousto-optic response elicited by a high-intensity ultrasound field during thermal therapy can be used to monitor the onset of lesion formation, ascertain lesion volume, and provide real-time control of exposure duration.
\end{abstract}

Keywords-Ultrasound, Optical imaging, Ultrasoundmodulated optical tomography, Acousto-optic imaging, Photorefractive crystal, Turbid media, Tissue optics, High-intensity-focused ultrasound, Thermal therapy.

The optical field traversing an object can be modified through the application of a sound wave through a process known as the acousto-optic (AO) effect. In the case of optically transparent media, originally described in the early twentieth century, the sound wave creates density fluctuations within the material which,

Address correspondence to Todd W. Murray, Department of Mechanical Engineering, University of Colorado at Boulder, Boulder, CO, USA. Electronic mail: todd.murray@colorado.edu in turn, produce refractive index variations and diffract the optical field. ${ }^{3}$ In optically turbid media, the interaction is complicated by multiple scattering as the light follows a tortuous path through the sample. In addition to producing density variations, the sound field also causes scattering sites within the media to displace, leading to an additional mechanism through which the phase of the optical field is modulated at the ultrasound frequency. The AO effect is localized to the region of space where the light and sound interact, and the degree to which the optical field is modulated depends on the characteristics of the sound field as well as the optical and mechanical properties in the interaction region.

Acousto-optic imaging (AOI), also referred to as ultrasound-modulated optical tomography, makes use of the AO effect to image subsurface optical contrast (and in some cases mechanical contrast) in optically turbid media such as biological tissue. ${ }^{9,18,30,34,47}$ The basic concept behind this hybrid imaging approach is straightforward: a medium is subjected to both an ultrasound and optical field and, subsequent to the interaction, the scattered light is collected and the net phase or frequency shift imparted on the light is measured. The ultrasound is then moved to different regions of the sample and this process is repeated, such that an image of the ultrasound modulation strength can be constructed. The contrast in such an acoustooptic image is related to the optical properties of the interaction region. Areas of higher optical absorption, for example, produce a smaller ultrasound-modulated optical signal because less light is modulated within, and collected from, these regions. The primary advantage of AOI over purely optical techniques, such as diffuse optical tomography, ${ }^{14}$ for imaging turbid media comes in the spatial resolution. Ultrasound 
exhibits substantially lower scattering in biological tissue than light, and the interaction region or "sensing zone" in AOI can thus be reduced through focusing the ultrasound field, allowing for the measurement of optical contrast within biological tissue with the spatial resolution of ultrasound.

Acousto-optic imaging was originally proposed for biological imaging more than 20 years ago, and several reviews of the technique are available in the literature. $^{10,35,46,48}$ The primary technical challenge in AOI has been in the development of techniques to measure the AO signal with acceptable sensitivity. The ultrasound-modulated optical signal originates from the interaction region, which is typically quite small depending on the spatial resolution required. The un-modulated light presents a strong background in which this small signal must be detected. Moreover, the phase modulation imparted on the light by the ultrasound is spatially random in that it varies from point to point in a given detection region. The interference between modulated and un-modulated light external to the sample produces a speckle pattern that is intensity modulated. The individual speckles are not, however, modulated in phase, such that simply collecting these speckles into a single optical detector leads to a reduction in modulation depth and low signal-to-noise ratio. In biological tissue, the speckle pattern is also time variant due to physiological motion, and therefore any detection system suitable for in vivo application must be able to accommodate this speckle decorrelation. ${ }^{15}$ Several detection techniques have been developed to overcome these difficulties including multi-detector approaches based on a CCD camera, ${ }^{31,52}$ photorefractive crystal (PRC) based interferometry, ${ }^{36,38,39,51}$ and spectral filtering of the modulated photons using a Fabry-Perot interferometer, ${ }^{40,41}$ or spectral hole burning. ${ }^{32}$ While a detailed description of all of these approaches is beyond the scope of this article, each has been successfully employed to image inclusions in biological tissue or tissue phantoms showing optical absorption, and in some cases scattering, contrast with the surrounding media. The detection approach that will ultimately yield the highest signal-to-noise ratio for in vivo imaging applications, however, remains an open question.

Acousto-optic imaging allows one to detect optical contrast within the imaged region, but the relationship between this contrast and the actual optical properties is not straightforward. AOI thus typically provides qualitative information. In some cases, however, the AOI response may also be used in a quantitative sense to measure the optical properties in a localized tissue volume or to track changes in optical properties in response to a therapeutic process. In the former case, AOI may find application in supplementing conventional ultrasound imaging, ${ }^{4}$ and potentially improve the specificity of ultrasound in distinguishing benign from malignant tumors. In the latter case, AOI may be used to monitor and guide ablative tumor therapy. In this article, we first describe the origin of the AO response and review approaches that have been proposed to obtain quantitative measures of tissue optical properties. We then present experimental results demonstrating that the local optical transport mean free path in scattering media can be obtained by varying the pressure of the ultrasound field and processing the resulting $\mathrm{AO}$ response, a technique referred to as pressure contrast imaging (PCI). ${ }^{27,28}$ Finally, we describe a recently developed approach for controlling high-intensityfocused ultrasound (HIFU) therapy using the AO response elicited by the HIFU field itself to track thermally induced changes in the tissue optical properties. ${ }^{25,26}$

\section{Quantifying the Acousto-Optic Response}

A schematic of a basic AO sensing or imaging system is shown in Fig. 1. The laser source is typically continuous wave $(\mathrm{CW})$, ideally operating in the nearinfrared region of the spectrum in the so-called biological window where optical penetration into soft biological tissue is maximized. ${ }^{11}$ More recently, pulsed lasers with a pulse width longer than the acoustic transient time through the region of interest in the sample have also been employed. ${ }^{39,40}$ Such laser sources allow for an increase in the optical fluence incident on the sample surface and subsequent increase in the signal-to-noise ratio. The ultrasound source pumping the AO response is shown perpendicular to the optical axis. Both the $\mathrm{CW}$ and pulsed ultrasound sources have been used, and pulsed sources, being somewhat preferable in that resolution, along the acoustic axis on the order of the spatial pulse length of the ultrasound wave packet can be readily achieved. The detection system is shown in a trans-illumination configuration, on the opposite side of the sample from the laser source. The detection system serves to demodulate the light scattered from the sample, converting the phase or frequency modulation imparted by the ultrasound on the light to an intensity modulation at a photodetector or CCD array for detection and subsequent processing.

For optically turbid media, light entering the sample is multiply scattered, with some photons traversing a path taking them from the entrance point, through the ultrasound interaction region, and on to the detection point. Other photons follow a similar tortuous path from source to detection point, but bypass the interaction region, and the remaining majority of photons are scattered away from the detection aperture or absorbed within the specimen volume. As the light travels from one scattering site to another, phase shifts
152

153

154

155

156

157

158

159

160

161

162

163

164

165

166

167

168

169

170

171

172

173

174

175

176

177

178

179

180

181

182

183

184

185

186

187 


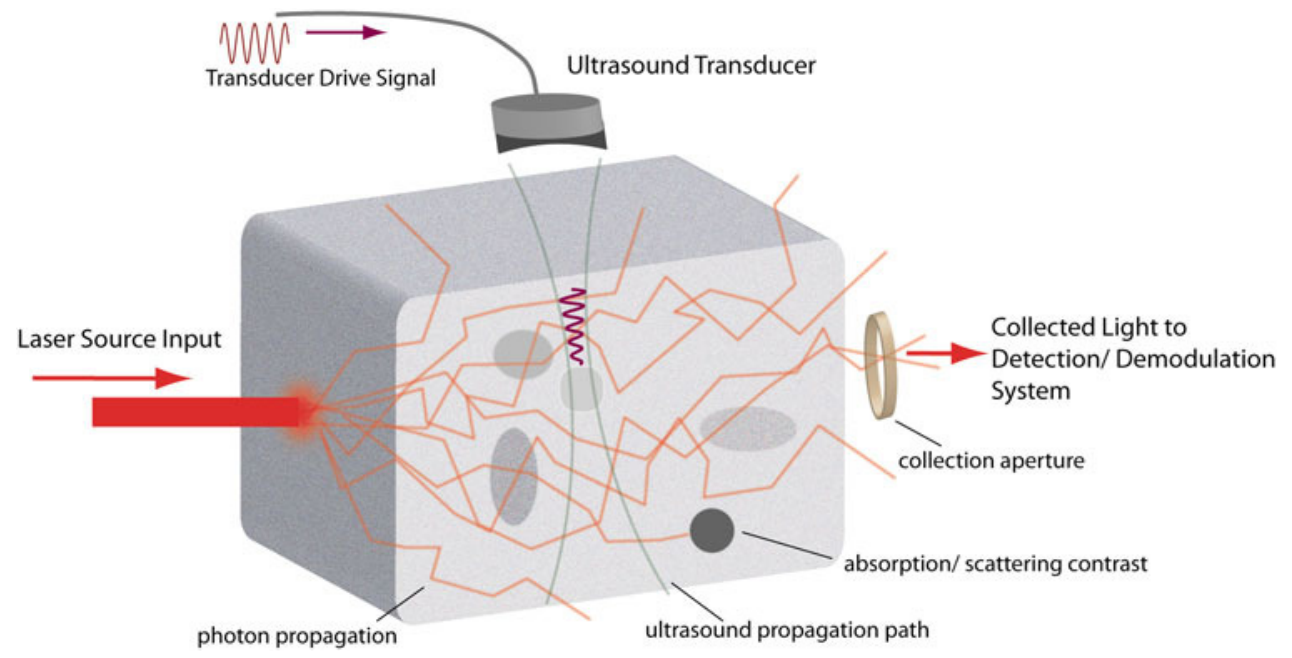

FIGURE 1. A schematic of a basic AO sensing and imaging systems.

are accumulated. In the presence of ultrasound, the phase is modified by (a) ultrasound induced changes in the refractive index $\Delta \varphi_{n, K}$ the phase change associated with refractive index changes over free path $K$; and (b) ultrasound induced displacement of optical scatterers $\Delta \varphi_{d, K}$, the phase shift associated with scattering event $K$. The net accumulated phase shift over each propagation path is then found by summing the phase shifts as the light propagates through the sample. Theoretical models for each of the modulation mechanisms have been described in the literature, and implemented in Monte Carlo (MC) simulations for various sample and ultrasound source configurations. ${ }^{18,30,42,44,45}$ Referring back to Fig. 1, the AO signal received at the detection point depends on the optical properties over the path from the source point to the interaction region, the interaction of ultrasound and light within the interaction region, the optical properties along the propagation path from the interaction region to the detection point, and the characteristics of the detection scheme itself. For a known ultrasound field, and in the absence of acoustic heterogeneity, the net phase shift imparted on the light reaching the interaction region can be related to the optical absorption and reduced scattering coefficients of the region. However, in the absence of a full model-based inversion algorithm, it is in general not possible to extract these parameters directly from the measured AO signal. While implementation of the inverse problem in AOI has not yet been demonstrated, improvements in MC simulation speed may ultimately make this approach feasible. ${ }^{29}$

There have been limited demonstrations of quantitative AOI presented in the literature, focusing primarily on the measurement of the optical absorption coefficient. Kim et al. ${ }^{22}$ used an AOI configuration in which cylindrical absorbing inclusions were embedded in a scattering background, the ultrasound axis was aligned along the axis of the cylinders, and the optical axis was aligned perpendicular to this direction. Here, CCD-based detection was used, where a reduction in speckle contrast in the presence of ultrasound is used to quantify the AO interaction. The authors normalized the $\mathrm{AO}$ response by measurements outside of the cylinders and were able to relate this normalized response to the absorption coefficient of the cylinders using the Beer-Lambert law. Using two optical wavelengths, they were also able to measure the total concentration and concentration ratio of two dyes with different spectral characteristics. Bratchenia et al. ${ }^{5,6}$ used a related experimental approach applied to more complex geometries. In this study, a normalized AO signal is obtained by measuring the $\mathrm{AO}$ response through the region of interest with and without the presence of an absorber. A calibration measurement is used to determine the relationship between absorption coefficient and AO response at a single point in the sample. The authors demonstrate the ability to map absorption coefficients in 3D using this approach. They also demonstrate the ability to measure total dye concentrations and ratio of dye concentrations by applying the technique at two optical wavelengths. This study shows great promise and demonstrates that AOI can potentially be applied for functional imaging to determine, for example, total hemoglobin content and oxygen saturation in tissue.

In addition to the techniques mentioned above, it is possible to quantify the optical properties in a given region through measurement of the AO response at multiple pressures. An advantage of this approach is that the measurement is independent of the optical path to the interaction region, and from the interaction region to the detection system. It is also possible 
to use the temporal changes in the AO signal to non261 invasively determine the onset of thermal lesion for262 mation and obtain a quantitative measure of lesion size 263 created during HIFU therapy. We present an overview 264 of our recent study in these areas in the remainder of this article. All experimental measurements were made using a PRC-based detection system. We therefore begin by describing this detection approach and how it is employed for each of these measurements.

\section{MATERIALS AND METHODS}

\section{Photorefractive Crystal Based Detection}

Photorefractive crystal-based interferometry has been widely employed for laser-based ultrasonic inspection applications. ${ }^{8}$ More recently, it has been adopted by several groups for AOI applications. ${ }^{12,36,38,39,51}$ We note that there are several configurations that have been reported for PRC detection, and here we focus only on the approach developed and used by our group. ${ }^{25-28,36}$ A schematic of the PRCbased AOI system is illustrated in Fig. 2. A continuous wave $\mathrm{Nd}$ :YAG $(\lambda=1064 \mathrm{~nm})$ laser is sent through a variable beam splitter where it is divided into a signal beam and a reference beam. The signal beam passes through a beam expander and the transparent walls of a water filled test tank, and on to the sample surface where the fluence is approximately $I=200 \mathrm{~mW} / \mathrm{cm}^{2}$. Scattered light transmitted through the sample is collected and sent to the PRC. The reference beam is expanded to $I=15 \mathrm{~mW} / \mathrm{cm}^{2}$ and directed around the test tank and on to the PRC. A $7 \times 7 \times 7 \mathrm{~mm}$ GaAs $\mathrm{PRC}$ was used for these experiments. The signal and reference beams interfere at the PRC, creating a complex intensity grating. In the PRC, this intensity grating is converted to an index of refraction grating through the photorefractive effect. The reference beam diffracts from this grating in a process known as two-wave mixing, and this diffracted reference beam has a wavefront matching that of the signal beam. A 2.1-kHz, 5-kV (peak to peak) electric field is applied across the PRC to enhance the two-wave mixing process. The PRC can be thought of as an adaptive holographic recording media, with the index of refraction grating rewritten in response to intensity changes on a time scale corresponding to the characteristic response time of the crystal. The response time is a function of the fluence incident on the crystal and is approximately $10-15 \mathrm{~ms}$ in our experimental configuration. The PRC is configured such that the diffracted reference beam is in-phase with the transmitted signal beam, such that these beams interfere constructively at the photodetector. The output of the photodetector is sent to a data acquisition system.

The sound source is a $1.1-\mathrm{MHz}$ HIFU transducer with a more or less "cigar shaped" focal zone $2.0 \mathrm{~mm}$ in diameter and $9.0 \mathrm{~mm}$ long, measured along the acoustic axis. In the absence of ultrasound, the collected signal and transmitted reference beams interfere constructively at the PRC, and a maximum intensity is observed at the photodetector. The presence of ultrasound modulates the phase of the light passing through the interaction region at the ultrasound frequency. As the PRC response time is much slower than the period of the ultrasound, the crystal cannot respond. This results in a wave front mismatch between the transmitted signal beam and diffracted reference beam. The constructive interference between these two beams is compromised, leading to a net reduction in the intensity observed at the photodetector. As an ultrasound pulse propagates through the sample, the timedependent intensity at the photodetector is recorded and converted to a one-dimensional spatial scan of the AO response through the sample using the speed of sound. It can be shown that the intensity change at the photodetector arising from the ultrasound modulation is given by Blonigen et al. ${ }^{2}$

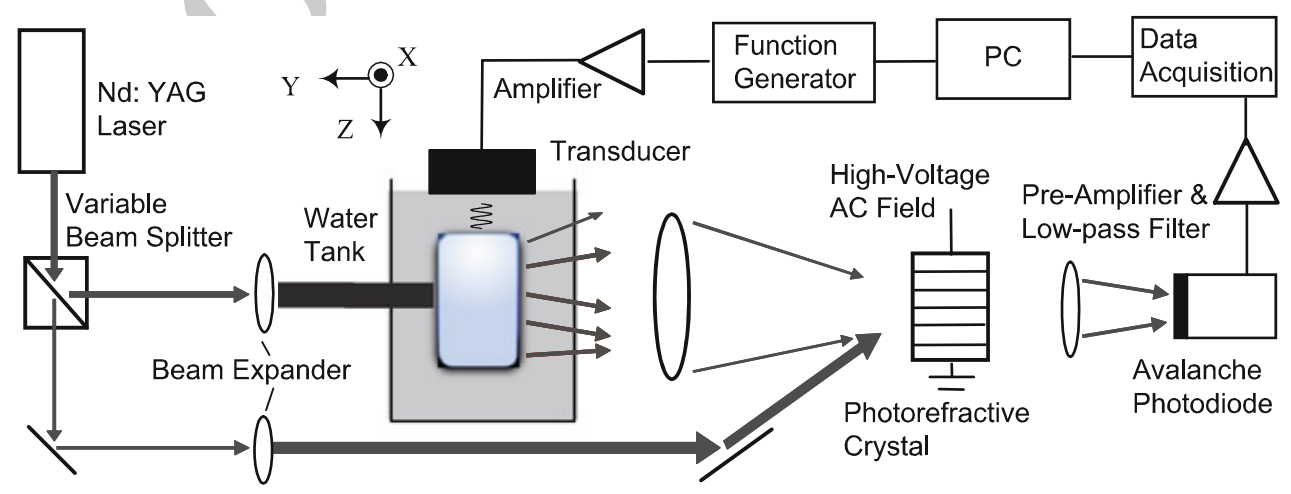

FIGURE 2. Experimental setup for PRC based detection of AO signals. ${ }^{27}$ 


$$
I_{\mathrm{s}}(t)=\chi(\eta(z, t)) v(\alpha, \gamma)\left\{\frac{1}{n} \sum_{j=1}^{n}\left(1-J_{0}\left(\left|\Phi_{j}\right|\right)\right)\right\} .
$$

340

341

342

343

344

345

346

347

348

349

350

351

352

353

354

355

356

357

358

359

360

361

362

363

364

365

366

367

368

369

370

371

373

375

376

377

378

379

380

381

382

383

384

385

386 where $\chi$ is a constant; $\eta(z, t)$ gives the amount of modulated light reaching the detection system, which depends on both the amount of light reaching the interaction region and the optical path from the interaction region to the detection system; $v(\alpha, \gamma)$ characterizes the detection efficiency of the PRC and is a function of the absorption within the crystal $\alpha$ and the complex gain; $\gamma$, and $n$ is the number of optical paths through the sample. The phase shift $\Phi_{j}$ gives the accumulated phase shift over path $j$ and can be related to the ultrasound pressure amplitude through $\Phi_{j}=\beta P_{j}$ where $\beta$ is a function of the transport mean free path $l_{t}$. The transport mean free path is related to the optical absorption $\left(\mu_{\mathrm{a}}\right)$ and reduced scattering $\left(\mu_{\mathrm{s}}^{\prime}\right)$ coefficients through: $l_{\mathrm{t}}^{-1}=\mu_{\mathrm{s}}^{\prime}+\mu_{\mathrm{a}}$. In tissues where scattering dominates absorption, the transport mean free path is given by the reciprocal of the reduced scattering coefficient.

\section{Pressure Contrast Imaging}

In PCI, the dependence of the AO signal on ultrasound pressure amplitude is used to gain insight into the net phase shift imparted on the light by the ultrasound in the interaction region, and to ultimately measure the transport mean free path within this region. In this technique, $\mathrm{AO}$ signals are detected at two different ultrasound drive pressure amplitudes. ${ }^{27,28}$ As the pressure pulse profile changes as the ultrasound pulses traverse the focal region, only the $\mathrm{AO}$ signals measured at the times at which the pulses reach the focus are considered. The focal pressures are referred to as $P^{\mathrm{f} 2}$ and $P^{\mathrm{f} 1}$. Referring back to Eq. (1), the pressure contrast signal (PCS) is defined as

$$
\mathrm{PCS}=\frac{\sum_{j=1}^{n}\left(1-J_{0}\left(\left|\beta P_{j}^{\mathrm{f}}\right|\right)\right)}{\sum_{j=1}^{n}\left(1-J_{0}\left(\left|\beta P_{j}^{\mathrm{f} 1}\right|\right)\right)}
$$

If the experimental setup remains stationary between measurements, then the PCS is only dependent on the pressure pulses used and the local optical properties of the interaction region. The first three terms in Eq. (1), associated mainly with light propagation outside of the interaction region and the detection process itself, cancel. We note that PCS imaging relies on the higher-order terms in the detection response. If we consider a single optical path and expand the Bessel functions, then it is apparent that the terms of the second order must be used to retain the dependence of PCS on $\beta$. The sensitivity of PCS to changes in $\beta$ is maximized when one pressure pulse is as high as possible, while the other is as low as possible. In the former case, one is limited by safety considerations, while in the latter, one is limited by signalto-noise ratio. The system is calibrated to determine the PCS, for a given set of pressure pulses, as a function of transport mean free path. Once this is accomplished, the transport mean free path of any region in a sample can be determined, regardless of optical heterogeneities in the media surrounding the probed position.

In our experiments, we use three-cycle ultrasound pulses with amplitudes of $P^{\mathrm{fl}}=0.3 \mathrm{MPa}$ and $P^{\mathrm{f} 2}=1.5 \mathrm{MPa}$. In order to minimize changes in the detection system associated with ambient vibrations, the pulses are sent to the sample in rapid sequence, separated in time by $97 \mu$ s. The PCS is then determined by dividing the AO signal measured when the 1.5 and $0.3 \mathrm{MPa}$ pulses reach the focal region. Tissue phantoms fabricated from polyacrylamide were used for the PCS experiments, with the reduced scattering coefficient controlled through the addition of polystyrene spheres and absorption coefficient selected by adding India ink. All samples were $40 \times 25 \times 40 \mathrm{~mm}^{3}$ along the $x, y$, and $z$ axes, respectively. Unless otherwise noted, inclusions fabricated within the phantoms all had dimensions of $6 \times 6 \times 20 \mathrm{~mm}^{3}(x, y, z)$. The ultrasound transducer was positioned such that the focus was at the center of the inclusions. The relatively long dimension along the $z$-axis (ultrasound axis) was chosen such that the entire three-cycle ultrasound wave packet was within an inclusion at the time when the PCS was measured.

\section{Monitoring Therapeutic Response}

The optical setup and transducer position used for HIFU monitoring are identical to that used for PCI. However, the transducer drive and signal processing on the detection side are somewhat different as illustrated in Fig. 3a. The figure shows the essential features of the system necessary to understand the approach, and further details can be found in the literature. $^{25,26}$ The HIFU transducer is driven by sequential pulse trains at $1.1-\mathrm{MHz}$ generated by amplitude modulating a $1.1 \mathrm{MHz}$ sine wave with a $50-\mathrm{Hz}$ square wave. As each ultrasound pulse train passes through the interaction region, the AO signal is reduced, while in the absence of a pulse train, the AO signal returns to the baseline level. The AO signal is thus periodic at the ultrasound modulation frequency. Figure $3 \mathrm{~b}$ shows an example of an $\mathrm{AO}$ signal detected in ex vivo chicken breast using this approach. In order to increase the sensitivity of detection, the AO signal output from the photodiode is fed into a lock-in amplifier (LIA). The integration time and roll-off of
418 


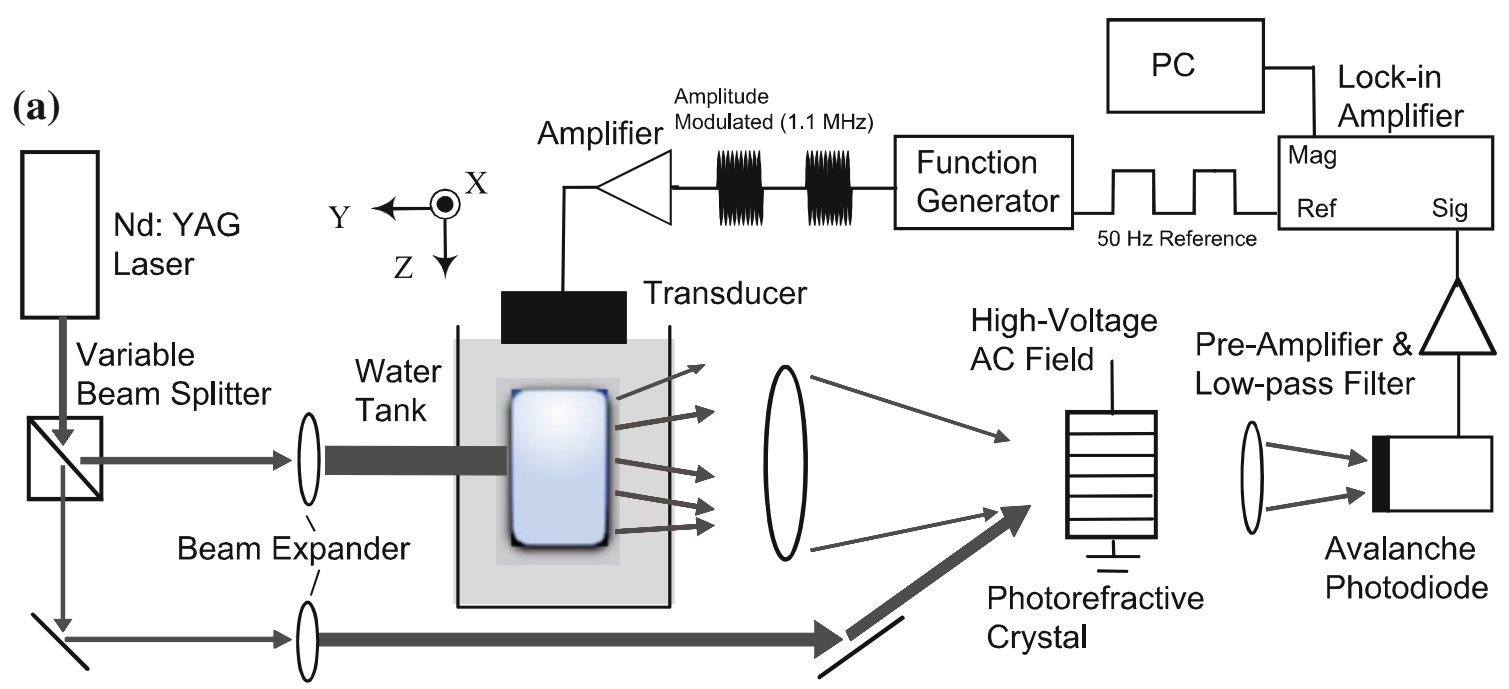

(b)

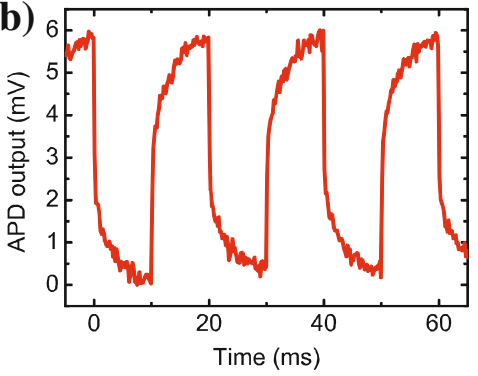

(c)

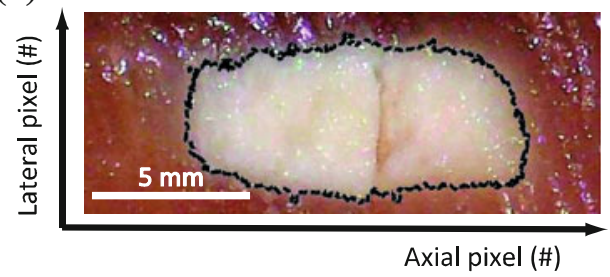

FIGURE 3. (a) Experimental setup for AO monitoring of HIFU therapy using lock-in detection. (b) Representative time domain signal at the photodetector (signal averaged 1000 times). (c) A cross section of a lesion formed in ex vivo chicken breast.

the LIA were set at $30 \mathrm{~ms}$ and $12 \mathrm{~dB} /$ octave, respectively, and the reference signal for the LIA was derived from the square wave used to amplitude modulate the HIFU drive. The magnitude of the AO response, measured as a function of time through the LIA, was then output to a personal computer to detect and track the progression of lesion formation in the focal zone.

The samples used in the HIFU study were ex vivo chicken breast purchased at a local store $24 \mathrm{~h}$ before experiments. The samples were cut to a size of $50 \times 50 \mathrm{~mm}$ along the $x$ and $z$ axes and $15-30 \mathrm{~mm}$ along the $y$-axis (see Fig. 3a). They were degassed in $1 \%$ phosphate buffered saline solution for $3 \mathrm{~h}$. The samples were held in freshly degassed water in the test tank with an acrylic holder that provided weak compression along the $y$-axis. Two or three lesions were typically formed in each sample, with a minimum spacing between lesions of $10 \mathrm{~mm}$ along the $x$-axis. After lesion formation, the samples were cross-sectioned and digital images of the lesion were taken. The lesion volume was estimated from these images using an edge detection algorithm and assuming cylindrical symmetry. Figure $3 \mathrm{c}$ shows one such image of a lesion and the lesion borders determined through the algorithm. Samples found to contain large blood vessels in the HIFU focal region were not included in the reported results.
The baseline AOI signal amplitude, detected through the LIA, varied from point to point in a given sample and between samples. Each signal, $\Delta S(t)$, was thus normalized to the value obtained early in the experiment $S_{0}$ before lesion formation. The normalized change in AOI signal during the exposure $\Delta S_{\mathrm{n}}\left(t_{\mathrm{f}}\right)$ is given as

$$
\Delta S_{\mathrm{n}}\left(t_{\mathrm{f}}\right)=\left|\frac{\Delta S(t)}{S_{0}}-1\right| \times 100 \%,
$$

where $t_{\mathrm{f}}$ indicates the time at which exposure ends.

\section{RESULTS}

\section{Pressure Contrast Imaging}

Figure 4a shows the AO signal amplitude as a function of ultrasound pressure in a homogeneous tissue phantom with a reduced scattering coefficient of $7 \mathrm{~cm}^{-1}$. The signal amplitude shown was measured at the time when the ultrasound wave packet crossed the focal point of the transducer. The pressures used for PCI are also indicated on the figure. Figure $4 \mathrm{~b}$ shows the measured AOI signal measured in response to three-cycle ultrasound tone bursts at 0.3 and $1.5 \mathrm{MPa}$, separated in time by $97 \mu$ s in the same phantom. 

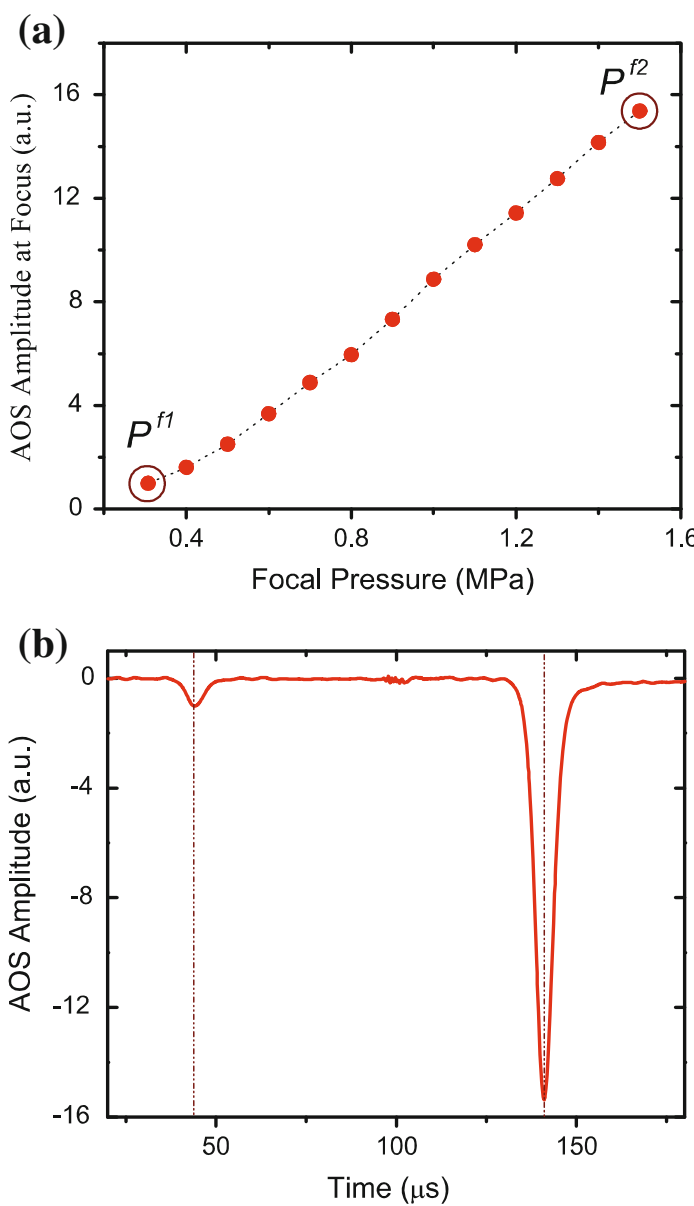

FIGURE 4. (a) AO signal amplitude at the focal position as a function of ultrasound drive pressure. The drive pressures used for $\mathrm{PCl}$ are given by the circles. (b) AO signal amplitude for two ultrasound pulses of peak pressure 0.3 and $1.5 \mathrm{MPa}$ separated in time by $97 \mu \mathrm{s}$. The times when each ultrasound pulse reaches the focus are given by the vertical lines. ${ }^{27}$

The dips in the signal correspond to the ultrasound pulses traversing the illuminated region of the phantom, and the width of these dips corresponds roughly to the transient time through this region. The ultrasound wave packets cross the focal region at approximately 45 and $142 \mu \mathrm{s}$, respectively. It is the value of the AO signal recorded at the two times, which is used to calculate the PCS at the focus through Eq. (2).

We measured the PCS at the center of several homogeneous phantoms with different reduced scattering coefficients in order to calibrate the system. The results are shown as the circles in Fig. 5. The abscissa is given in terms of inverse transport mean free path, where $l_{\mathrm{t}}^{-1}=\left(\mu_{\mathrm{a}}+\mu_{\mathrm{s}}^{1}\right)$. In order to reduce the uncertainty in the measurement, the data taken from each sample were averaged 10,000 times and stored on the computer. This process was then repeated 80 times. The error bars on the plot indicate the standard error in the measurements. The squares in Fig. 5 show the PCS measured in phantoms with embedded inclusions

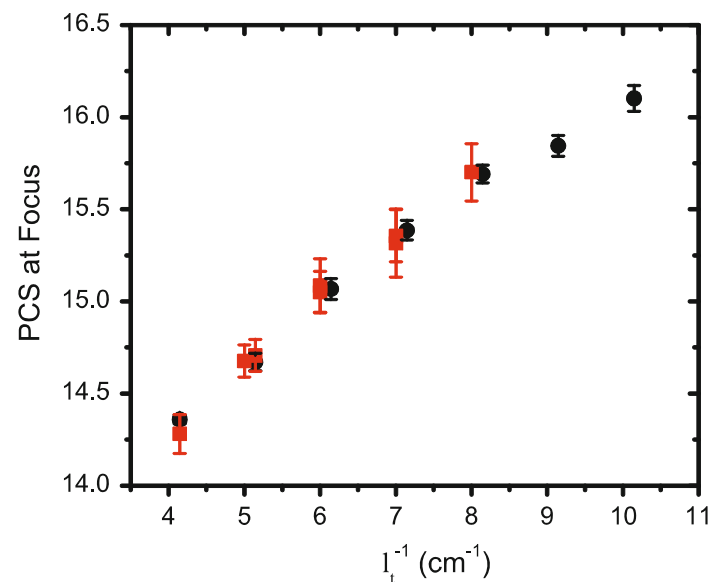

FIGURE 5. PCS measures at the focal position as a function of transport mean free path for homogeneous scattering phantoms (circles) and phantoms with inclusions showing absorption contrast with the surrounding media (squares). ${ }^{24}$

having absorption contrast with the surrounding 506 media. ${ }^{24}$ The eight measurements were taken in 507 phantoms with a reduced scattering coefficient of $4.0 \quad 508$ and $5.0 \mathrm{~cm}^{-1}$. The inclusions in the $4.0 \mathrm{~cm}^{-1}$ phan- 509 toms had the following properties: $\left(\mu_{\mathrm{s}}^{\prime}\right)=4.0 \mathrm{~cm}^{-1} \quad 510$ and $\mu_{\mathrm{a}}=0.14,1.0,2.0,3.0 \mathrm{~cm}^{-1}$, while those in the 511 $5.0 \mathrm{~cm}^{-1}$ phantom were $\left(\mu_{\mathrm{s}}^{\prime}\right)=5 \mathrm{~cm}^{-1}$ and $\mu_{\mathrm{a}}=0.14, \quad 512$ $1.0,2.0,3.0 \mathrm{~cm}^{-1}$. In all measurements, the transducer 513 focus was placed at the center of the inclusions. $\quad 514$

Figure 6 shows a two-dimensional scan of a scat- 515 tering inclusion with $\left(\mu_{\mathrm{s}}^{\prime}\right)=10 \mathrm{~cm}^{-1}$ embedded in a 516 phantom with a background $\left(\mu_{\mathrm{s}}^{\prime}\right)=7 \mathrm{~cm}^{-1}$. The size 517 of the inclusion was $5 \times 6 \times 20 \mathrm{~mm}^{3}(x, y, z)$. The 518 transducer focus was again positioned at the center of 519 the inclusion and the phantom was scanned in the $x-y \quad 520$ plane. The measurement parameters are the same as 521 those given above, but the signal averaging used for 522 each measurement was reduced by a factor of 10 . The 523 scanning step size was $0.5 \mathrm{~mm}$, and the measured data 524 were smoothed and interpolated to take into account 525 the finite beam width of the transducer. The color scale 526 shows the measured PCS, and this value can be con- 527 verted to $l_{\mathrm{t}}^{-1}$ using the data shown in Fig. 5.

\section{HIFU Monitoring}

529

Figure $7 \mathrm{a}$ shows the magnitude of the normalized AO responses measured during HIFU exposure of a chicken breast sample for different time periods. The measurements were taken at different positions within the same sample, with the same peak intensity of $3300 \mathrm{~W} / \mathrm{cm}^{2}$. The exposure times were 5,30 , and $40 \mathrm{~s}$, as shown on the figure, and the signals from each exposure duration are offset for comparison. Images of cross sections of the lesions created during each exposure are shown in Fig. 7b. For each of the exposures,
530 531 532 533 534 535 536 537 538 539 
540 the AO signal was observed to increase rapidly to the 541 baseline value within the first second. During the first $5425 \mathrm{~s}$ of exposure, the signal remained near the baseline 543 value. For the longer exposures, the value then began 544 to reduce with the end of exposure values given by 0.45 545 for the 30-s exposure and 0.28 for the 40-s exposure. 546 No lesion was detected for the 5-s exposure, while 547 lesion volumes of 250 and $470 \mathrm{~mm}^{3}$ were measured for

the 30- and 40-s exposures, respectively.

In order to assess the dependence of the change in AO response $\Delta S_{\mathrm{n}}$, as given by Eq. (3), on lesion volume, measurements were performed with a wide variety of ultrasound exposure times and peak pressures. The composite results from the measurements are plotted in Fig. 8, where the legend indicates the ultrasound parameters used. Measurements on different samples, but with the same ultrasound exposure parameters, produced lesions with marked differences in volume. However, $\Delta S_{\mathrm{n}}$ showed a monotonic increase with lesion volume.

Given the strong correlation between $\Delta S_{\mathrm{n}}$ and lesion volume, the ability to control lesion volume based on the $\mathrm{AO}$ response was explored. In these experiments,

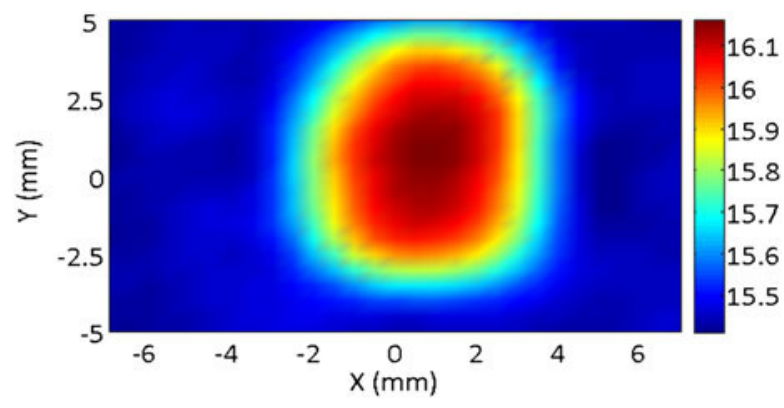

FIGURE 6. A 2D pressure contrast image of a scattering inclusion of $\left(\mu_{\mathrm{s}}^{\prime}\right)=10 \mathrm{~cm}^{-1}$ in a background media of $\left(\mu_{\mathrm{s}}^{\prime}\right)=7 \mathrm{~cm}^{-1}$. The color scale shows the amplitude of the PCS. $^{24}$

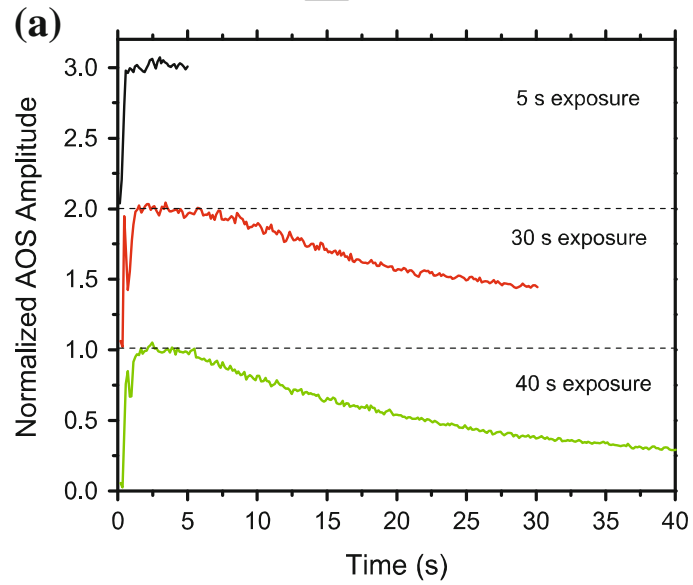

a given $\Delta S_{\mathrm{n}}$ was selected, and the HIFU exposure continued until this value was reached, after which the lesion volume was determined. These measurements were then compared to lesions produced with fixed exposure durations. For these measurements, 157 exposures were made with the same peak intensity $\left(3300 \mathrm{~W} / \mathrm{cm}^{2}, 10 \mathrm{MPa}\right)$ with the exposure duration controlled in real time through the AO response. An additional 187 exposures were made using several preset exposure durations. The mean and standard deviations of the resulting lesion volumes are shown in Fig. 9 as a function of mean exposure duration. A 30$40 \%$ reduction in the standard deviation of the lesion volume was observed when using AO feedback control when comparing lesions of similar size.

\section{DISCUSSION}

Pressure contrast imaging and HIFU monitoring are two AOI-based approaches in which the measured

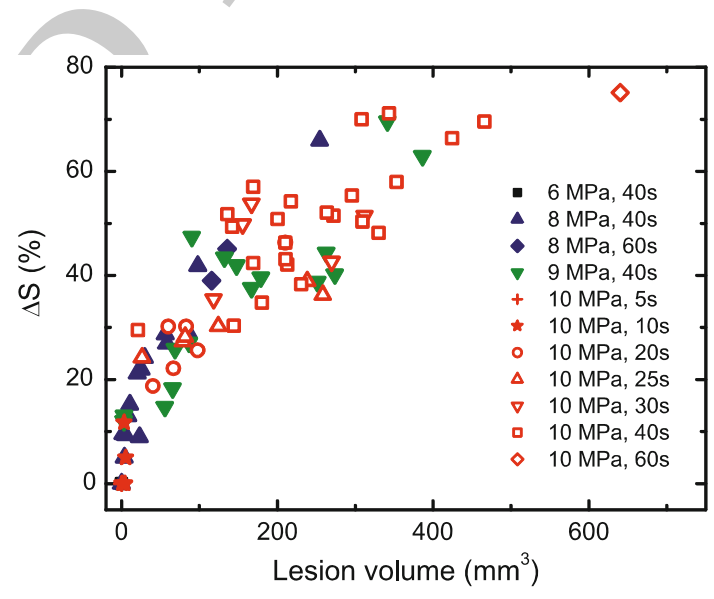

FIGURE 8. AOS signal amplitude reduction as a function of lesion volume for a wide range of HIFU exposure conditions. ${ }^{26}$

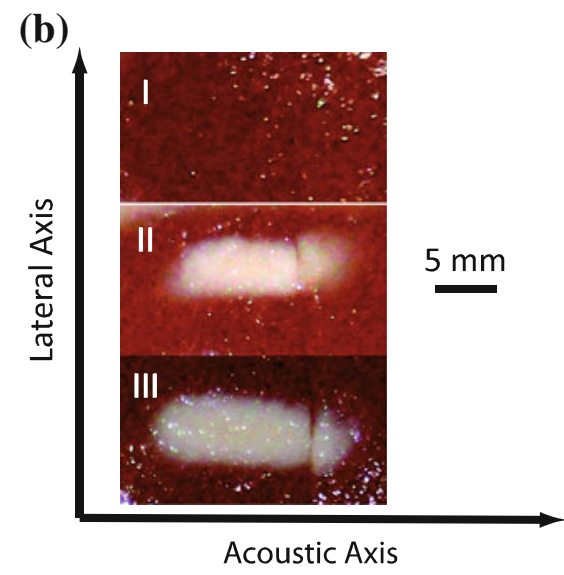

FIGURE 7. (a) Normalized AOS amplitude as a function of HIFU exposure time. The 30- and 5-s exposure signals are offset for ease of viewing, and (b) photographs of the lesions produced by: I. 5-s exposure, II. 30-s exposure, and III. 40-s exposure. ${ }^{26}$ 


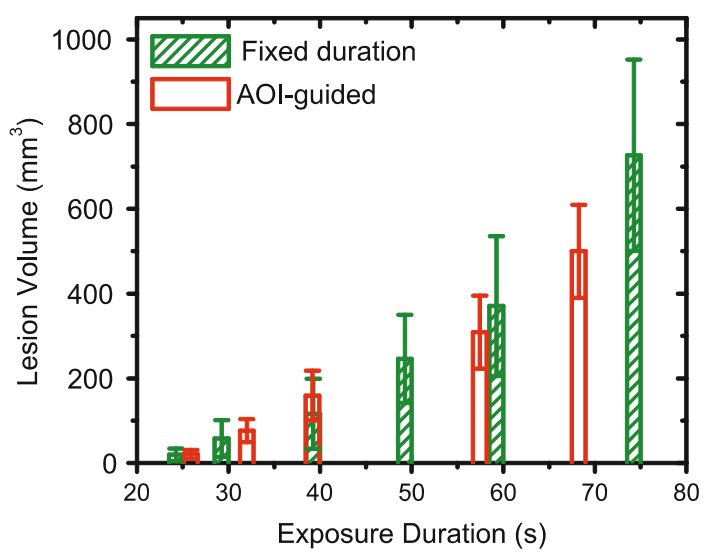

FIGURE 9. A plot of lesion volumes as a function of mean exposure time for exposures controlled by monitoring the AOS and lesions created under fixed-exposure conditions. The error bars give the standard deviation of the lesion volumes. $^{26}$

581 582 583 584
AO response can be directly related to a physical parameter associated with the interaction region. In the case of PCI, the optical transport mean free path can be measured, while the HIFU monitoring experiments indicate that the extent of lesion can be estimated. The experimental results and potential applications of these techniques are discussed shortly.

\section{Pressure Contrast Imaging}

The PCS results shown in Fig. 5 require further discussion. In the case of homogeneous scattering samples, we find that the PCS increases nearly linearly with reduced scattering coefficient. Furthermore, an increase in the PCS corresponds to a decrease in the net phase shift imparted on the light: i.e., the net phase shift decreases as the reduced scattering coefficient is increased. While this may be somewhat counterintuitive, it is in agreement with other measurements and has been reported to be associated with an anticorrelation between the two mechanisms of ultrasound modulation of light. ${ }^{23}$ The sensitivity of PCI to reduced scattering makes it as a potentially attractive approach for measuring this quantity in biological tissue or tissue phantoms. This is also an advantage of this technique, and AOI in general, over photoacoustic tomography, which is primarily sensitive to optical absorption. ${ }^{50}$ The PCS results for the absorbing and scattering inclusions show good agreement with the homogeneous phantoms indicating that (a) the transport mean free path is the key parameter controlling the PCS response for a given set of pressure pulses, and (b) the PCS is somewhat unaffected by the optical properties of the media surrounding the interaction zone, thus providing a localized measurement. In other experiments, we have also found that the PCS is relatively insensitive to the point within the optical field, where the measurement is taken: particularly, for samples with high reduced scattering coefficients. In a phantom with reduced scattering coefficient of $7 \mathrm{~cm}^{-1}$, for example, we found the PCS to be constant to within the experimental error as the transducer was scanned $12.0 \mathrm{~mm}$ along, and perpendicular to, the optical axis in a homogeneous scattering phantom. The AOI response, which primarily tracks the intensity of the optical field, varied approximately $30 \%$ over this same region. ${ }^{24}$

The main drawback of PCI comes in the sensitivity, and stems from the fact that the origin of the effect is in the higher-order terms of the detection response. The data shown in Fig. 5 required 800,000 measurements, and 80,000 measurements were used at each point to obtain the image shown in Fig. 6. We used a pulse repetition rate of $4 \mathrm{kHz}$, yielding a minimum of 200 and $20 \mathrm{~s}$ for the two sets of data. The number of measurements required in a given application is dependent on the resolution required in the measurement of $l_{\mathrm{t}}$. Taking the acoustic transient time across the illumination region to be approximately $30 \mu \mathrm{s}$, and considering that two pulses must cross this region in each measurement, one could increase the measurement speed by a factor of $\sim 4$ operating at a higher pulse repetition rate. Further improvements are possible through increasing the sensitivity of the detection system using, for example, a long-pulse laser. ${ }^{39,40}$ Due to this limitation, we expect that, while 2D PCI is possible as illustrated in Fig. 5, it is not particularly attractive as an imaging technique, but rather may find application as a local sensing technique. Suspect regions of tissue found during ultrasound mammography, for example, may be characterized at a single point or small region using PCI as a non-invasive probe. The combination of the data acquired from both ultrasound and PCI could be used to improve the specificity of the approach. A second application area may be in monitoring tumor response to treatment over time.

\section{HIFU Monitoring}

High-intensity-focused ultrasound is a promising technique to create thermal damage or tissue necrosis in localized regions of soft tissues, with applications including the treatment of tumors in liver, prostate, breast, and kidneys. ${ }^{13,19,20,43}$ The ultrasound intensity required to create a given therapeutic effect can, however, be difficult to estimate a priori due to the inhomogeneous nature of tissue and cooling effects associated with blood flow. ${ }^{33}$ It is therefore advantageous to develop real-time techniques to monitor lesion formation in tissue and potentially provide 
668 feedback to guide the treatment process. While MRI ${ }^{17}$ 669 and ultrasound imaging ${ }^{49}$ are currently used clinically 670 for this purpose, they each have limitations for real671 time monitoring in terms of cost and image acquisition 672 speed (MRI) or the limited sensitivity with which 673 lesions can be detected in the absence of cavitation 674 (ultrasound imaging). ${ }^{16}$ AOI is a particularly promis675 ing alternate technique for this application; the HIFU 676 field itself can be used to drive the AO interaction, with 677 the high pressures producing large AO signals. More678 over, the optical properties of tissue have been shown 679 to vary markedly in between normal and necrotic tis680 sues, with necrotic tissue showing increased absorption 681 and scattering and the specific increase dependent on 682 tissue type. ${ }^{1,21,37}$

683 The HIFU monitoring results demonstrate that 684 real-time monitoring of lesion formation and growth is 685 possible using AOI combined with lock-in detection. 686 In the experimental results shown in Fig. 7, the AO 687 signal amplitude is shown to decrease in response to 688 lesion formation, indicating an increase in the optical 689 scattering or absorption coefficient. This is consistent 690 with the changes in optical properties of soft tissues 691 reported in the literature. ${ }^{1,21,37}$ In the experimental 692 results given in Fig. 8, the normalized change in AO 693 signal magnitude $\Delta S_{\mathrm{n}}$ was observed to be highly cor694 related with lesion volume under a wide variety of 695 exposure conditions, with $\Delta S_{\mathrm{n}}$ increasing monotoni696 cally with lesion volume. Lesion volume depends on a 697 number of factors including the HIFU field charac698 teristics and exposure duration, as well as the physical 699 properties of the tissue along the ultrasound propa700 gation path. Although the data in this study were 701 collected from similar tissue samples, the lesion yol- lesion formation than B-mode ultrasound in the absence of boiling. A key finding in this study was that the AOI response can be monitored in real time and used to control the extent of lesion. This is shown in Fig. 9, comparing the lesion volumes created under fixed exposure conditions with AO-guided exposures. The data are plotted as a function of mean exposure time. The standard deviation of the measured lesion volume, given by the error bars on the plot, is the key metric of interest. The standard deviations for both the fixed and AO-guided exposures were observed to increase with lesion volume. The AO-guided exposures, however, show a significantly smaller standard deviation (30-40\%) when comparing with lesions of similar volume.

The normalized change in AO signal amplitude has been shown to be an effective indicator of lesion volume, and is relatively insensitive to HIFU exposure parameters and sample geometry. In this study, sample thicknesses along the optical axis ranged from 15 to $30 \mathrm{~mm}$, resulting in large differences in the magnitude of the direct AO response measured across samples. The normalized change in the AO response, however, is effectively localized to the region of the sample where the optical properties are being modified and insensitive to the amount of light reaching the interaction region (source geometry) or the amount of modulated light collected and processed (detection geometry). Once the normalized change in AOI response is calibrated for a given HIFU source and tissue type, it can potentially provide a powerful means of determining and controlling extent of lesion. Furthermore, this technique may find application in non-invasively guiding other types of thermally ablative therapy, such as laser and microwave.

\section{CONCLUSIONS}

The interaction of diffuse light and ultrasound results in a modulation in the phase of the light at the ultrasound frequency. In the case of bounded acoustic beams (either collimated or focused), only those photons that traverse the beam experience this modulation, with the strength of modulated light depending on several factors, including the local light intensity, the optical absorption coefficient, and the reduced scattering coefficient within the interaction volume. Thus, by monitoring the intensity of modulated light, one can ascertain the optical properties of diffusive media (such as tissue) with the spatial resolution of ultrasound, which is typically on the order of a millimeter.

Quantitative AOI is confounded by the multiple parameters that impact the $\mathrm{AO}$ response. However, if one considers the ratio of the measured AO signal
758 
obtained at two acoustic pressure amplitudes (all else being equal), then terms dependent on the local light intensity and the light propagation path cancel out, and one is left with a convenient way to measure the optical properties at a point in space. This technique, termed PCI, suffers from low signal-to-noise ratio and thus cannot be practically employed to generate $2 \mathrm{D}$ images. However, one could use the technique to probe specific locations in tissue, providing pointwise information that could be combined with conventional ultrasound imaging to yield enhanced diagnostic capability.

A principal challenge in high-intensity-focused ultrasound therapy is monitoring the onset of lesion formation and ascertaining lesion volume. Variability associated with the repeated use of fixed-exposure parameters can be substantial, particularly in highly vascularized, heterogeneous tissues. By using the HIFU beam to pump the AO response, one has a means for assessing in real time the changing optical properties of the tissue in the HIFU focal region, which is precisely where the lesion forms. One can determine when the lesion starts to grow and estimate the volume based on the percentage change in the AO response. One can also use the $\mathrm{AO}$ signal as a simple feedback control parameter to control HIFU exposure duration. Such feedback control, when applied to the formation of multiple lesions in excised chicken breast, resulted in more predictable lesion generation than that could be achieved using fixed-exposure parameters. We expect that the advantages of this approach will become even more pronounced in vivo, where spatially heterogeneous perfusion will induce even greater variability in lesion size and shape.

\section{ACKNOWLEDGMENTS}

The authors would like to acknowledge the generous financial support of the Bernard M. Gordon Center for Subsurface Sensing and Imaging Systems under National Science Foundation Award No. EEC9986821.

\section{REFERENCES}

${ }^{1}$ Ben-David, M., R. Cantor, N. Balbul, M. Yehuda, and I. Gannot. Measuring tissue heat penetration by scattered light measurements. Lasers Surg. Med. 40:494-499, 2008. ${ }^{2}$ Blonigen, F. J., A. Nieva, C. A. DiMarzio, S. Manneville, L. Sui, G. Maguluri, T. W. Murray, and R. A. Roy. Computations of the acoustically induced phase shifts of optical paths in acoustophotonic imaging with photorefractive-based detection. Appl. Opt. 44:3735-3746, 2005.

${ }^{3}$ Born, M., and E. Wolf. Principles of Optics. Cambridge: Cambridge University Press, 1999.

${ }^{4}$ Bossy, E., L. Sui, T. W. Murray, and R. A. Roy. Fusion of conventional ultrasound imaging and acousto-optical sensing by use of a standard pulsed ultrasound scanner. Opt. Lett. 30:744-746, 2005.

${ }^{5}$ Bratchenia, A., R. Molenaar, and R. P. H. Kooyman. Feabilility of quantitative determination of local optical absorbances in tissue-mimicking phantoms using acoustooptic sensing. Appl. Phys. Lett. 92:113901, 2008.

${ }^{6}$ Bratchenia, A., R. Molenaar, T. G. van Leeuwen, and R. P. H. Kooyman. Millimeter-resolution acousto-optic quantitative imaging in a tissue model system. J. Biomed. Opt. 14:034031, 2009.

${ }^{7}$ Coussios, C., C. H. Farny, G. T. Haar, and R. A. Roy. Role of acoustic cavitation in the delivery and monitoring of cancer treatment by high-intensity focused ultrasound (HIFU). Int. J. Hyperth. 23:105-120, 2007.

${ }^{8}$ Dewhurst, R. J., and Q. Shan. Optical remote measurement of ultrasound. Meas. Sci. Technol. 10:R139-R168, 1999.

${ }^{9}$ Dolfi, D., and F. Micheron. Imaging process and system for transillumination with photon frequency marking. International Patent WO 98/00278, 1989.

${ }^{10}$ Elson, D. S., R. Li, C. Dunsby, R. Eckersley, and M.-X. Tang. Ultrasound-mediated optical tomography: a review of current methods. Interface Focus 1:632-648, 2011.

${ }^{11}$ Fantini, S., and M. A. Franceschini. Frequency domain techniques for tissue spectroscopy and imaging. In: Handbook of Optical Biomedical Diagnostics, edited by V. V. Tuchin. Bellingham, WA: SPIE Press, 2002, pp. 405-453.

${ }^{12}$ Farahi, S., G. Montemezzani, A. Grabar, J. Huignard, and F. Ramaz. Photorefractive acousto-optic imaging in thick scattering media at $790 \mathrm{~nm}$ with a $\mathrm{Sn}_{2} \mathrm{P}_{2} \mathrm{~S}_{6}$ :Te crystal. Opt. Lett. 35:1798-1800, 2010.

${ }^{13}$ Gelet, A., J. Y. Chapelon, R. Bouvier, O. Rouviere, Y. Lasne, D. Lyonnet, and J. M. Dubernard. Transrectal high-intensity focused ultrasound: minimally invasive therapy of localized prostate cancer. J. Endourol. 14:519528, 2000.

${ }^{14}$ Gibson, A. P., J. C. Hebden, and S. R. Arridge. Recent advances in diffuse optical imaging. Phys. Med. Biol. 50:R1-R43, 2005.

${ }^{15}$ Gross, M., P. Goy, B. C. Forget, M. Atlan, F. Ramaz, A. C. Boccara, and A. K. Dunn. Heterodyne detection of multiply scattered monochromatic light with a multipixel detector. Opt. Lett. 30:1357-1359, 2005.

${ }^{16}$ Hill, C. R., and G. R. ter Haar. High intensity focused ultrasound-potential for cancer treatment. Br. J. Radiol. 68:1296-1303, 1995.

${ }^{17}$ Hynynen, K., W. R. Fruend, H. E. Cline, A. H. Chung, R. D. Watkins, J. P. Vetro, and F. A. Jolesz. A clinical, noninvasive, MR imaging-monitored ultrasound surgery method. Radiographics 16:185-195, 1996.

${ }^{18}$ Kempe, M., M. Larionov, D. Zaslavsky, and A. Z. Genack. Acousto-optic tomography with multiply scattered light. J. Opt. Soc. Am. A 14:1151-1158, 1997.

${ }^{19}$ Kennedy, J. E. High intensity focused ultrasound in the treatment of solid tumors. Nat. Rev. Cancer 5:321-327, 2005.

${ }^{20}$ Kennedy, J. E., F. Wu, G. R. ter Haar, F. V. Gleeson, R. R. Philips, M. R. Middleton, and D. Cranston. High
824

825

826

827

828

829

830

831

832

833

834

835

836

837

838

839

840

841

842

843

844

845

846

847

848

849

850

851

852

853

854

855

856

857

858

859

860

861

862

863

864

865

866

867

868

869

870

871

872

873

874

875

876

877

878

879

880

881

882

883

884

885

886

887 
intensity focused ultrasound for the treatment of liver tumors. Ultrasonics 42:931-935, 2004.

${ }^{21}$ Khokhlova, T. D., I. M. Pelivanov, O. A. Sapozhniko, V. S. Solomatin, and A. A. Karabutov. Opto-acoustic diagnostics of the thermal action of high-intensity focused ultrasound on biological tissues: the possibility of its applications and model experiments. Quantum Electron. 36:1097-1102, 2006.

${ }^{22} \mathrm{Kim}, \mathrm{C}$., and L. H. V. Wang. Multi-optical-wavelength ultrasound modulated optical tomography: a phantom study. Opt. Lett. 32:2285-2287, 2007.

${ }^{23}$ Kothapalli, S. R., S. Sakadzic, C. Kim, and L. V. Wang. Imaging optically scattering objects with ultrasound-modulated optical tomography. Opt. Lett. 32:2351-2353, 2007.

${ }^{24}$ Lai, P. Photorefractive Crystal-Based Acousto-Optic Imaging in the Near-Infrared and Its Applications. Ph.D. Dissertation, Boston University, Boston, MA, 2011.

${ }^{25}$ Lai, P., J. R. Mclaughlan, A. B. Draudt, R. O. Cleveland, R. A. Roy, and T. W. Murray. Monitoring and guidance of high intensity focused ultrasound exposures in real time using acousto-optic imaging: feasibility and demonstration ex vivo. Proc. SPIE 7564:75642B, 2010.

${ }^{26}$ Lai, P., J. R. McLaughlan, A. B. Draudt, T. W. Murray, R. O. Cleveland, and R. A. Roy. Real-time monitoring of high-intensity focused ultrasound lesion formation using acousto-optic sensing. Ultrasound Med. Biol. 37:239-252, 2011.

${ }^{27}$ Lai, P., R. A. Roy, and T. W. Murray. Quantitative characterization of turbid media using pressure contrast acousto-optic imaging. Opt. Lett. 34:2850-2852, 2009.

${ }^{28}$ Lai, P., R. A. Roy, and T. W. Murray. Sensing the optical properties of diffusive media by acousto-optic pressure contrast imaging. Proc. SPIE 7177:71771G, 2009.

${ }^{29}$ Leung, T. S., and S. Powell. Fast Monte Carlo simulations of ultrasound-modulated light using a graphics processing unit. J. Biomed. Opt. 15:055007, 2010.

${ }^{30}$ Leutz, W., and G. Maret. Ultrasonic modulation of multiply scattered light. Physica B 204:14-19, 1995.

${ }^{31}$ Leveqe-Fort, S., A. C. Boccara, M. Lebec, and H. SaintJalmes. Ultrasonic tagging of photon paths in scattering media: parallel speckle modulation processing. Opt. Lett. 24:181-183, 1999.

${ }^{32}$ Li, Y., P. Hemmer, C. Kim, H. Zhang, and L. V. Wang. Detection of ultrasound-modulated diffuse photons using spectral-hole burning. Opt. Express 16:14862-14864, 2008.

${ }^{33}$ Maleke, C., and E. E. Konofagou. Harmonic motion imaging for focused ultrasound (HMIFU): a fully integrated technique for sonication and monitoring of thermal ablation in tissues. Phys. Med. Biol. 53:1773-1793, 2008.

${ }^{34}$ Marks, F. A., H. W. Tomlinson, and G. W. Brooksby. A comprehensive approach to breast cancer detection using light: photon localization by ultrasound modulation and tissue characterization by spectral discrimination. Proc. SPIE 1888:500-510, 1993.

${ }^{35}$ Murray, T. W., and R. A. Roy. Illuminating sound: imaging tissue optical properties with ultrasound. Acoustics Today 3:17-24, 2007.
${ }^{36}$ Murray, T. W., L. Sui, G. Maguluri, R. A. Roy, A. Nieva, F. J. Blonigen, and C. A. DiMarzio. Detection of ultrasound-modulated photons in diffuse media using the photorefractive effect. Opt. Lett. 29:25092511, 2004.

${ }^{37}$ Nilsson, A. M. K., C. Sturesson, D. L. Liu, and S. Andersson-Engels. Changes in spectral shape of tissue optical properties in conjunction with laser-induced thermotherapy. Appl. Opt. 37:1256-1267, 1998.

${ }^{38}$ Ramaz, F., B. C. Forget, M. Atlan, and A. C. Boccara. Photorefractive detection of tagged photons in ultrasound modulated optical tomography of thick biological tissues. Opt. Express 12:5469-5474, 2004.

${ }^{39}$ Rousseau, G., A. Blouin, and J. P. Monchalin. Ultrasoundmodulated optical imaging using a powerful long pulse laser. Opt. Express 16:12577-12590, 2008.

${ }^{40}$ Rousseau, G., A. Blouin, and J. P. Monchalin. Ultrasoundmodulated optical imaging using a high-power pulsed laser and a double-pass confocal interferometer. Opt. Lett. 34:3445-3447, 2009.

${ }^{41}$ Sakadzic, S., and L. V. Wang. High resolution ultrasoundmodulated optical tomography in biological tissues. Opt. Lett. 29:2770-2772, 2004.

${ }^{42}$ Sakadzic, S., and L. V. Wang. Modulation of multiply scattered coherent light by ultrasound pulses: an analytical model. Phys. Rev. E 72:033620, 2005.

${ }^{43}$ ter Haar, G. R., and C. Coussios. High intensity focused ultrasound: physical principles and devices. Int. J. Hyperth. 23:89-104, 2007.

${ }^{44}$ Wang, L. V. Mechanisms of ultrasound modulation of multiply scattered coherent light: an analytic model. Phys. Rev. Lett. 87:043903, 2001.

${ }^{45}$ Wang, L. V. Mechanisms of ultrasonic modulation of multiply scattered coherent light: a Monte Carlo model. Opt. Lett. 26:1191-1193, 2001.

${ }^{46}$ Wang, L. V. Ultrasound-mediated biophotonic imaging: a review of acousto-optical tomography and photo-acoustic tomography. Dis. Markers 19:123-138, 2003.

${ }^{47}$ Wang, L. V., S. L. Jacques, and X. Zhao. Continuous-wave ultrasonic modulation scattered light to image objects in turbid media. Opt. Lett. 20:629-631, 1995.

${ }^{48}$ Wang, L. V., and H. Wu. Biomedical Optics Principles and Imaging, Hoboken. New Jersey: Wiley, 2007.

${ }^{49}$ Wu, F., Z. Wang, W. Chen, J. Zou, J. Bai, H. Zhu, K. Li, F. Xie, C. Jin, H. Su, and G. Gao. Extracorporeal focused ultrasound surgery for the treatment of human solid carcinomas: early Chinese clinical experience. Ultrasound Med. Biol. 30:245-260, 2004.

${ }^{50} \mathrm{Xu}, \mathrm{M}$., and L. V. Wang. Photoacoustic imaging in biomedicine. Rev. Sci. Instrum. 77:041101, 2006.

${ }^{51} \mathrm{Xu}$, X., H. Zhang, P. Hemmer, D. K. Qing, C. Kim, and L. V. Wang. Photorefractive detection of tissue optical and mechanical properties by ultrasound modulated optical tomography. Opt. Lett. 32:656-658, 2007.

${ }^{52}$ Yao, G., and L. V. Wang. Theoretical and experimental studies of ultrasound-modulated optical tomography in biological tissue. Appl. Opt. 39:659-664, 2000. 